Chapter 5

\title{
Hematopoietic Stem Cells and Response to Interferon
}

\author{
Atsuko Masumi \\ Additional information is available at the end of the chapter \\ http://dx.doi.org/10.5772/54689
}

\section{Introduction}

Homeostasis in the bone marrow is dependent on the ability of hematopoietic stem cells (HSCs) to self-renew faithfully, differentiate into various lineages of the hematopoietic system, and form blood cells of several types (Figure 1) [1,2]. Under homeostatic conditions, HSCs are thought to be quiescent, and they are referred to as long-term reconstituting HSCs (LT-HSC) or dormant HSCs (dHSCs) [3,4]. Blood and immune cells are produced by the more differentiated short-term reconstituting HSCs (ST-HSCs) or multipotent progenitors (MPPs). Genetic and molecular studies of HSC self-renewal have identified candidate regulatory factors, including cell-intrinsic regulators, such as transcription factors and cell surface receptors, and cell-extrinsic regulators, such as the bone marrow niche and cytokines. Under certain conditions, such as inflammatory stress, HSCs differentiate into progenitor cells with less ability to self-renew, and they can be stimulated to divide and/or differentiate into all cell types in the peripheral blood $[5,6]$. Under inflammatory conditions, such as during bacterial infection or sepsis, an apparent expansion of lineage-negative Sca- $1+\mathrm{c}-\mathrm{Kit}+$ bone marrow cells (KSL) has been observed [7-11]. HSCs and progenitor cells are involved in the expansion of KSL; and expansion of the KSL population in the bone marrow has been associated with a loss of dormant LT-HSCs, reduced engraftment, and a bias towards myeloid lineage differentiation within that population. The process of the transition of HSCs from dormancy to activity is mediated by type I interferon (IFN) and type II IFN.

Interferon is produced by cells of the immune system in response to challenge by agents, such as viruses, bacteria, and tumor cells. Type I IFNs are induced by the genomes of many RNA viruses during viral infection, and they suppress viral replication and have immunomodulatory activity. IFNs are used clinically to treat viral diseases and malignancies, such as chronic myeloid leukemia (CML)[12]. In addition, under steady state conditions in the absence of infection, a small amount of intrinsic IFN is produced constitutively [13]. Recently, Essers et al. demonstrated that chronic activation of the IFN- $\alpha$ pathway impairs the function of HSCs and 
that acute IFN- $\alpha$ treatment promotes the proliferation of dHSCs in vivo. Studies exploring the application of type I IFN to target cancer stem cells are expected [14].

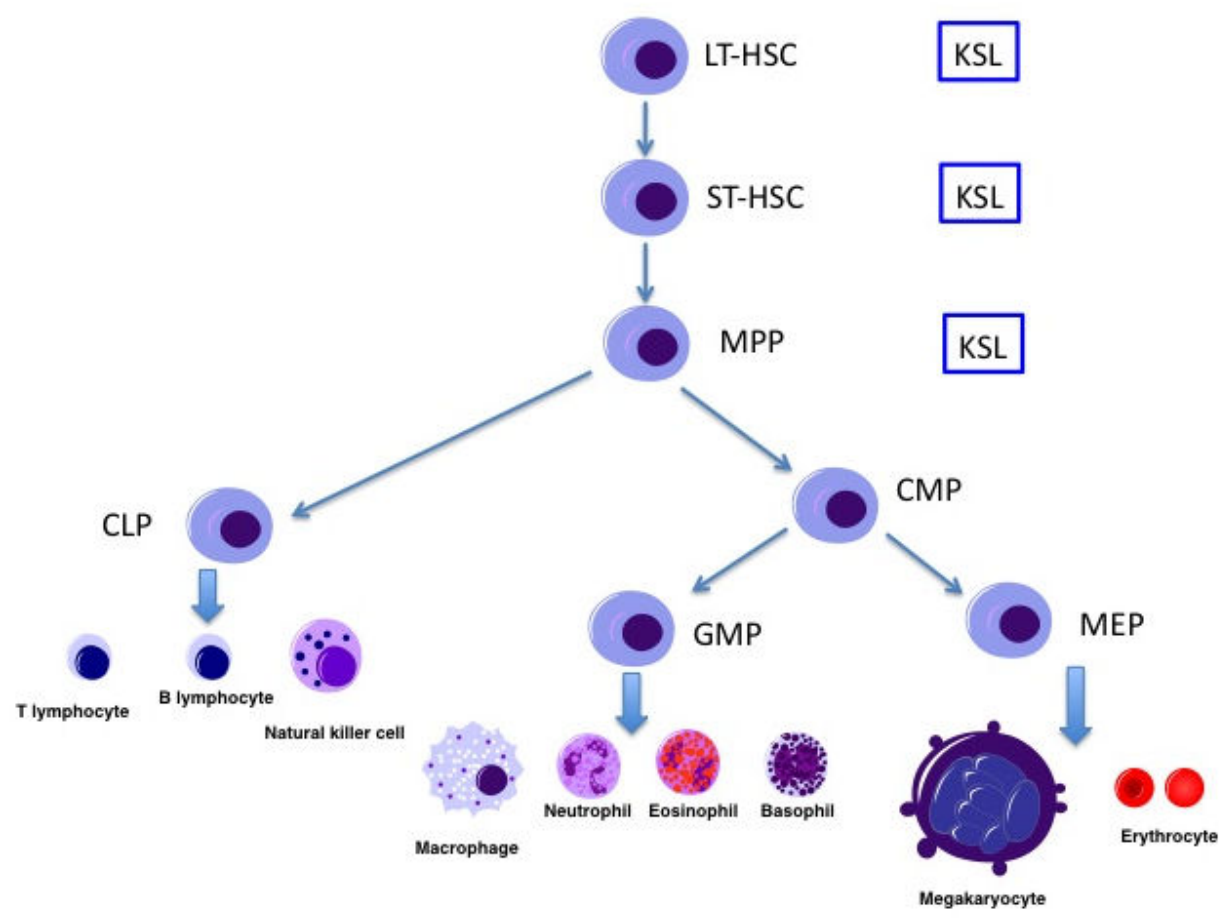

Figure 1. The fates of hematopoietic stem cells. HSCs are capable of self-renewal and differentiation into various lineages of the hematopoietic system to form all types of blood cells. LT-HSCS, ST-HSCs, and MPP are included in the KSL population.

Interferon regulatory factor-2 (IRF-2) is a typical inhibitor of the IFN response. Previously, I showed that IRF-2-/- mouse bone marrow cells contain an enhanced population of Sca-1-positive cells and reduced HSC activity in the KSL fraction [15]. Sato et al. reported that a marked reduction of hematopoietic stem cells in IRF-2-/- mice was dependent on a type I IFN-dependent mechanism [16]. In IRF-2-/- mouse bone marrow, type I IFN signalling is constitutively activated, and interferon-stimulated genes (ISGs) are continuously induced to activate cell cycle progression in HSCs. IRF-2 plays an important role enabling HSCs to maintain their inhibitory status against the type I IFN response.

Bone marrow HSCs are somatic stem cells that have a number of cell surface markers, which allow their prospective identification and isolation with FACS. Mouse models are usually used to examine HSC function in vivo. However, the HSC cell surface markers differ between mice and humans. Mouse HSCs in healthy adult bone marrow show a Lin-Sca-1+c-Kit+CD34- 
CD150+CD48- phenotype. In contrast, human HSCs are less well defined but present in the Lin-CD34+CD38- population within cord blood.

Infection or inflammation usually induces IFN production for host defense. Thus, the IFN response is a key factor in switching HSC activity to renewal in the hematopoietic system during the response to environmental conditions. This review will focus on the regulation of HSCs mediated by types I and II IFN.

\section{Hematopoietic stem cells in bone marrow}

Under homeostatic conditions, HSCs are thought to be quiescent, and they are referred to as long-term reconstituting HSCs (LT-HSC) or dormant HSCs (dHSCs) [3,4]. During homeostasis, dormant HSCs(dHSCs) are consistently inactive with residing bone marrow niches. Homeostatic HSCs (hHSCs) divide and self-renew to generate the progenitors [14]. dHSCs are almost in the G0 phase of cell cycle and found in niches within the cavities of trabecular bone. dHSCs are resistant to anti-proliferative chemotherapy such as 5-fluorouracil (5-FU). Homeostatic HSCs(hHSCs) divide and self-renew. dHSCs and hHSCs exit their niches and undergo selfrenewing divisions, followed by maintenance to homeostasis of hematopoietic cell populations. IFN- $\alpha$ induces cell cycle entry of dHSCs and hHSCs, resulting in rapidly dividing active HSCs (aHSC), which can produce progenitors and then differentiate ito mature cell types [14].

\section{Type I IFN (IFN- $\alpha)$}

IFNs play a critical role in the regulation of resistance to viral infection and activation of the innate and acquired immune system through IFN-stimulated genes (ISGs). IFNs also have an anti-malignant effect and are important intrinsic cytokines for host defense. To induce ISGs, type I IFN signaling is mediated by activation of the Jak-STAT signaling pathway through the type I IFN receptor (Figure 2). Many RNA viruses and double-stranded RNAs that mimic polyinosinic-polycytidylic acid (poly[I:C]) induce the expression of type I interferons (IFN- $\alpha$, IFN- $\beta$ ) that bind to the IFN $\alpha / \beta$ receptor (IFNAR), which mediates Jak-STAT signaling followed by ISG transcription. Treatment with IFN- $\alpha$ induces transcription and cell surface presentation of stem cell marker Sca-1, which is downstream of IFN- $\alpha$ signaling, on hematopoietic stem cells and progenitor cells [14].

The KSL population from IRF-2-/- mouse bone marrow is increased due to the enhancement of Sca-1. However, the CD150+CD48-KSL population is dramatically reduced because of a reduction of the CD150+ population in IRF-2-/- mouse bone marrow cells. IRF-2-/- HSCs are incapable of reconstituting lethally irradiated mice because of constitutive IFN signaling. Chronic exposure to IFN- $\alpha$ has a negative effect on HSC self-renewal. IFN- $\alpha$ induces the activation of the dormant HSC pool. Sato et al. indicated that chronic exposure of HSCs from IRF-2-/- mice to IFN- $\alpha$ resulted in a marked reduction of LT-HSCs and dHSCs[16]. This reduction of LT-HSC activity was rescued in IRF-2-/-IFNAR-/- dKO mice, but not completely, 
suggesting an IFN-independent mechanism exists in IRF-2-/- mice. Gene expression and transplantation analyses using HSCs from IRF-2-/-IFNAR-/- mice have demonstrated that IRF-2 regulates HSC populations independently of type I IFN signaling.

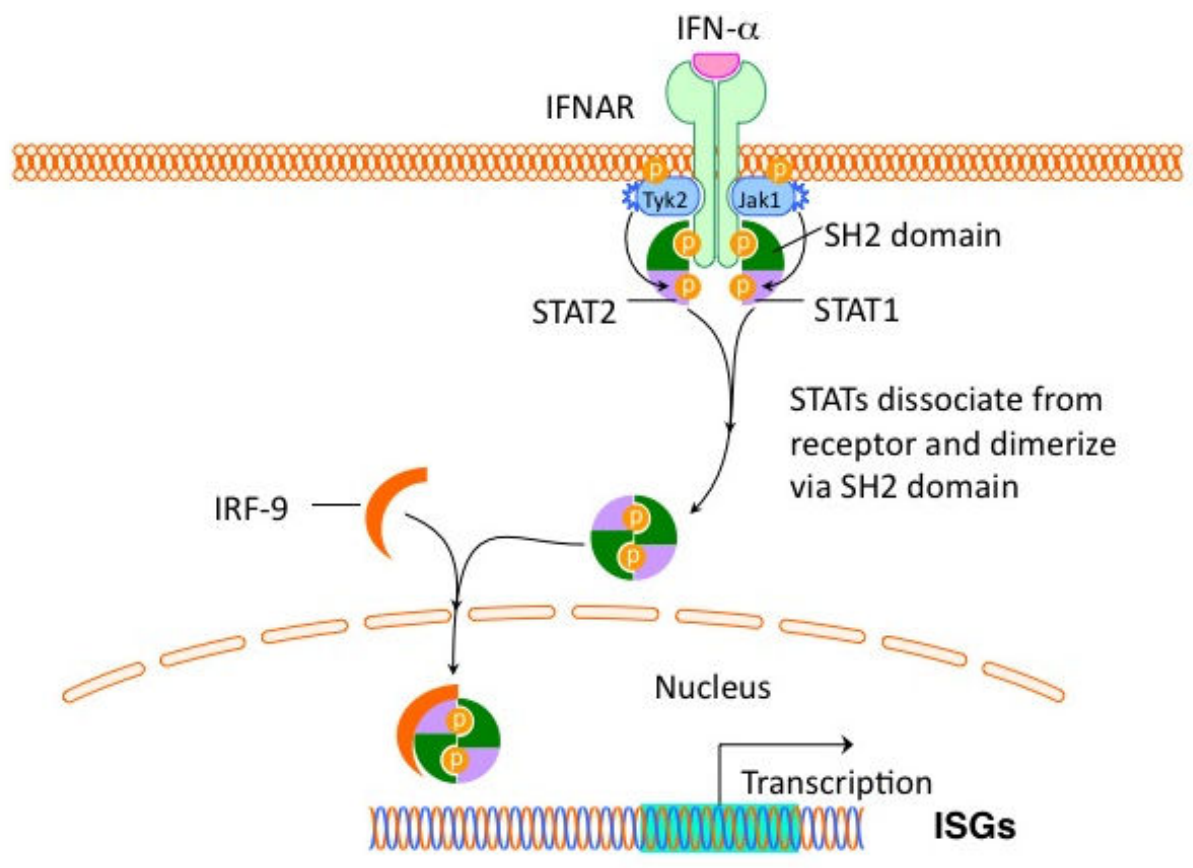

Interferon stimulated responsive element (ISRE)

Figure 2. Jak-Stat signaling pathway activated by Interferon. Type I IFN binds to the IFN receptor to stimulate the JakSTAT signaling pathway. Phosphorylated JAK1and Tyk2 phosphorylate STAT1 and STAT2, which heterodimerize via SH2 domain, and then translocated to nucleolus with IRF-9. The interferon-stimulated gene factor-3 (ISGF3) complex is transferred to the nucleus to bind to the ISRE consensus sequence that induces transcription, resulting in production of ISGs. STAT: Signal Transducers and Activator of Transcription, SH2:Src homology domain

A large quantity of whole bone marrow cells from IRF-2-/- mice can rescue recipients from lethal irradiation [15]. Whole bone marrow cells from IRF-2-/- mice were transplanted into lethally irradiated wild type mice, and KSL or CD150+CD48-Lin- cells from the recipient mice were isolated. Higher doses of bone marrow cells from IRF-2-/- mice could rescue recipients from lethal irradiation, even if the engraftment efficiency was poorer than that of wild-type mice. In addition, in the rescued recipients, the percentage of donor-derived KSL and CD150+CD48-Lin- cells from IRF-2-/- mice was higher compared to that of wild-type mice (Masumi et al., unpublished data).

The number of CD150+CD48-Lin- cells from IRF-2-/- mouse bone marrow present in a recipient wild-type environment is almost comparable to that of wild-type (Masumi et 
al., unpublished data). A marked reduction of CD150+CD48-Lin- cells in the bone marrow of IRF-2-/- mice may be due to the impairment of the stem cell niche in IRF-2-/mouse bone marrow. Essers et al. reported that wild-type HSC in chimeric mice with wild-type hematopoietic cells in an IFNAR-/- stromal environment are still efficiently activated by IFN- $\alpha$ and that IFN- $\alpha$ signaling is not required in the stem cell niche. The CD150+ population in KSL cells may be partly regulated by an IRF-2-dependent stem cell niche environment. In contrast, KSL populations from IRF-2-/- mouse bone marrow in the stromal environment of the recipient wild-type mice still show the phenotype of IRF-2-/- mice. CD150+CD48-KSL HSCs in untreated mice are predominantly in a quiescent intracellular Ki67-negative G0 phase. After injection of poly (I:C), most of these cells exit G0 and enter G1, activating the cell cycle. CD150+CD48-KSL in IRF-2-/- mice continuously enter an active cell-cycle state, which is dependent on chronic type I IFN signaling (Figure 3).

Homeostatic HSCs Dormant HSCS



Stem cell marker

Difficult self-renewing Homeostatic HSCs/IRF-2-/-
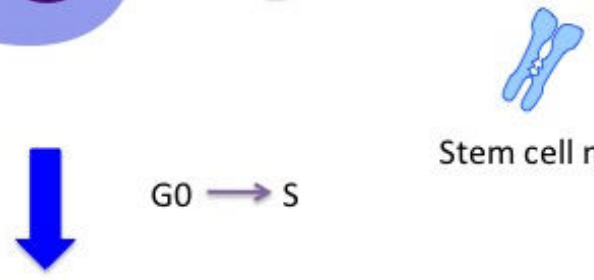

5-FU sensitive Cell death

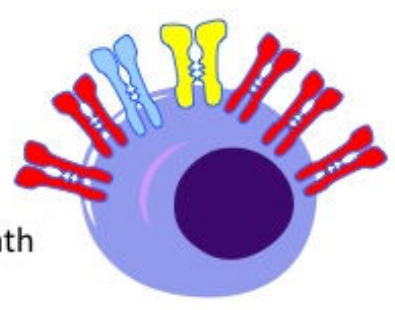

Activated HSC

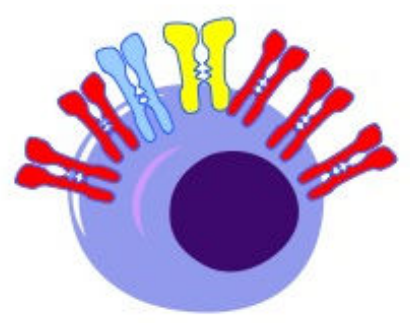

Progenitors

Figure 3. Chronic IFN stress in IRF-2-/- mice. In IRF-2-/- mice bone marrow cells, which cannot abrogate chronic IFN stimulation, Sca-1 is upregulated at the mRNA and protein levels, resulting in enhanced Sca-1 on the cell surface. However, CD150+CD48- cells are almost absent in the KSL of IRF-2-/- mouse bone marrow. Chronic IFN-a stimulation induces cell cycle entry of dormant and homeostatic HSCs as well as progenitors (MPPs). 
Most interestingly, several hematologic malignancies, including acute myeloid leukemia (AML) and chronic myeloid leukemia (CML), often contain a small population of malignant cells that show similarities in phenotype and function to normal HSCs. Dormant HSCs, which show the highest self-renewal potential of all HSCs and are almost permanently in the G0 phase of the cell cycle, are resistant to antiproliferative chemotherapy with agents, such as 5-FU. Cancer stem cells (CSCs) are resistant to radiation and antiproliferative chemotherapeutic agents because of their distinctive properties, which seem to be related to their stem cell-like character [17].

AML stem cells are reported to be located at the endosteal region of the bone marrow and are mainly noncycling. The tyrosine kinase inhibitor imatinib mesylate (Gleevec) blocks the constitutively active BCR-ABL kinase produced by the Philadelphia chromosome and is the first example of targeted chemotherapy as it acts against the causative mutation (BCR-ABL) that initiates the disease $[18,19]$. IFN- $\alpha$ was formerly a first-line treatment for $\mathrm{CML}$, with variable outcomes. Imatinib mesylate has replaced IFN- $\alpha$ owing to its better response rate and fewer side effects. However, intrinsic IFN- $\alpha$ may help the molecular agents that target cancer stem cells.

\section{Effect of IFN- $\gamma$ signaling in bone marrow}

IFN- $\gamma$ plays a critical role in innate immunity to intracellular pathogens, including the ehrlichiae, and is essential for host defense because of its ability to activate macrophages and promote Th1 responses. Many in vivo infectious studies have demonstrated that numerous pathogens induce production of IFN- $\gamma$, which mediates host defense. IFN- $\gamma$ is a critical defense against mycobacteria, as mice with a disrupted IFN- $\gamma$ receptor gene, IFNGR-1, did not respond to $M$. avium infection [20]; and human patients with mutations in the IFN- $\gamma$ receptor have died from disseminated M. avium infection [21]. However, a lesser known function of IFN- $\gamma$ is its ability to act as a moderator of hematopoiesis. Several studies have demonstrated that IFN- $\gamma$ inhibits formation of human bone marrow colonies and the growth of CD34+ bone marrow cells through differentiation and apoptosis [22-26]. In vitro, the addition of exogenous IFN$\gamma$ significantly inhibits myeloid and erythroid colony formation in both murine and human systems [22,27-30]. Zhao et al. demonstrated that IFN-is essential for the generation of a unique progenitor population that gives rise to mostly myeloid cells [31]. A colony-forming unit (CFU) assay indicated that treatment with IFN- $\gamma$ led to a reduction in the number of progenitor cells (Lin-c-Kit+Sca-1-) in bone marrow and enhanced them in the spleen [30]. Sorted KSL cells from IFN- $\gamma$-treated mice had greater potential for proliferation and were able to yield higher CFUs compared to control progenitor and KSL cells.

Genes induced by IFN- $\gamma$ are critical for HSC survival and maintenance via the expansion and proliferation of KSL [20,32]. This observation shows that IFN- $\gamma$ modulates the differentiation of hematopoietic progenitor cells, resulting in the enhanced production of mature blood cells. Investigations of mycobacterial infection have defined an important role for IFN- $\gamma$ in myelopoiesis [20,33-35]. Several bacterial and viral infection models have confirmed the effect of 
IFN- $\gamma$ on hematopoietic stem and progenitor cells (HSPCs). A murine in vivo infection model and in vitro human cell culture study showed that Escherichia coli stimulates the expansion and mobilization of hematopoietic progenitors (CD34-positive cells in humans and KSL cells in a murine system) [36-39]. Vaccinia virus infection increases immature myelocytes in peripheral blood [10]. These infections trigger the mobilization and differentiation of common myeloid progenitors. Intracellular bacterial infections cause the differentiation of hematopoietic progenitor cells through direct IFN- $\gamma$ signaling (Figure 4).

\section{IFN- $\gamma$ (Mycobacteria infection-induced)}



Figure 4. During infection, IFN- $\gamma$ is produced by several immune cells such as macrophages, NK cells, and lymphocytes. IFN- $\gamma$ activates HSCs and induces the expansion of MPPs.

Acute bacterial infection (Ehrlichia muris) induces myelopoiesis that is dependent on IFN$\gamma$ signaling. It has been demonstrated that infection-induced IFN- $\gamma$ acts on normally quiescent HSCs, leading to transient activation and promoting an expedited innate immune response $[7,8]$. Infection of mice with the malaria parasite Plasmodium chabaudi caused anemia and a decrease in erythrocytes. Malarial infection induces IL-7Ra+c-Kit ${ }^{\text {hi }}$ progenitors, which generate myeloid cells to decrease parasitemia in vivo. Lin-IL-7Ra+c-Kit ${ }^{\text {hi }}$ cells contribute to the clearance of malaria-infected erythrocytes in vivo. The infection of ifngr-/- mice with $P$. chabaudi did not increase the number of Lin-IL-7Ra+c-Kit ${ }^{\text {hi }}$ atypical progenitors [34]. Thus, IFN- $\gamma$ signaling is important for hematopoiesis and the innate immune system's response to acute infection.

Chronic mycobacterial infection also induces the proliferation of HSCs. M. avium infection seems to induce an increase in the proliferative fraction of primitive LT-HSCs and a substantial increase in the number of early-committed progenitors. IFN- $\gamma$ is strongly upregulated during M. avium infection. HSCs from Stat-1-/- and ifngr-/- mice infected with M. avium showed an impaired proliferative response by the LT-HSC population, indicating that the HSC response to $M$. avium infection is dependent on IFN- $\gamma$ signaling [33]. The effects of both in vitro and in vivo IFN- $\gamma$ treatment on KSL expansion were greatly reduced in Stat-1-/- and ifngr-/- mice compared to those in control mice [31]. Enhanced incorporation of BrdU into LT-HSCs from IFN- $\gamma$-treated mice and a modest impairment of the engraftment of whole bone marrow cells 
in IFN- $\gamma$-treated mice were detected [33]. IFN- $\gamma$ directly affects and modulates the proliferation and mobilization of HSCs.

\subsection{Human diseases and IFN- $\gamma$ signaling}

Human bone marrow failure syndromes, such as myelodysplastic syndromes (MDS), aplastic anemia (AA) and paroxysmal nocturnal hemoglobinuria (PNH) are characterized by a defect of stem and progenitor cell populations. The gene expression analysis of human CD34 cells treated with IFN- $\gamma$ indicated results that were similar to those of CD34 cells that are derived from patients with bone marrow failure syndromes, according to Zheng et al. [40]. IFN- $\gamma$ negatively modulates the self-renewal of CD34+CD38- stem cells and promotes differentiation of CD34+ cord blood cells expanded in vitro [26]. IFN- $\gamma$ is an important modulator of myeloid differentiation in myelocytopenias and pathogenesis.

IRF-2

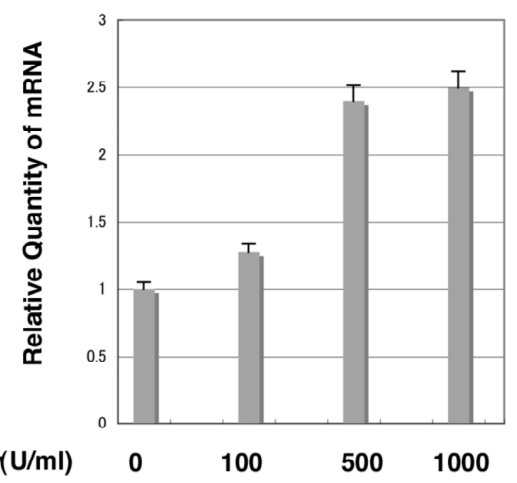

IRF-1



Figure 5. IFN- $\gamma$ stimulation induces expression of IRF-2 and IRF-1 in mouse hematopoietic stem/progenitor cells. Bone marrow cells from mice injected with 5 -FU $(150 \mathrm{mg} / \mathrm{kg})$ were cultured in the presence of IFN- $\gamma$ at the indicated concentrations. Real-time PCR was performed and the relative amounts of mRNA from the IRF-1 and IRF-2 genes were quantified with a $\beta$-actin primer [44].

\subsection{IFN- $\gamma$ signaling and megakaryopoiesis}

IFN- $\gamma$ has been reported to induce production of megakaryocytes and platelets [41,42]. A previous study reported that IFN- $\gamma$ accelerates megakaryocyte differentiation and IRF- 1 and IRF-2 are IFN- $\gamma$-inducible factors that enhance megakaryopoiesis [43-45]. Huang et al. reported that GATA-1 promotes megakaryopoiesis through the activation of IFN- $\gamma /$ STAT-1 signaling, of which IRF-1 is a downstream target. IFN- $\gamma$ enhanced colony formation and proliferation of megakaryocytes, but not their maturation [43]. When IFN- $\gamma$ was stimulated with bone marrow cells isolated from 5-FU-injected mice, IRF-2 expression was increased by around $80-100 \%$, but its induction level was much lower than that of IRF-1 (Fig. 5). Transduction of IRF-2 into mouse 
bone marrow stem/progenitor cells induces megakaryocyte differentiation, as we demonstrated with a colony formation assay [44]. Upregulation of IRF-2 by IFN- $\gamma$ treatment leads to CD41 expression, resulted in megakaryopoiesis [44]. IRF-2 plays an important role for hematopoiesis mediated by each type I and type II IFN response. However, the number of peripheral blood platelets is comparable between control mice and mice transplanted with IRF-2-transduced bone marrow stem cells/progenitors. IRF-2 enhances megakaryocyte colony formation, not its maturation, suggesting its cooperation with other factors for maturation [44, 45].

\section{Conclusion}

HSCs might be driven to exhaustion by IFN stimulation. IFN- $\gamma$ modulates HSCs differentiation into progenitors for host defense against extrinsic pathogen, inflammation, and other immune responses. IFN- $\alpha / \beta$ stimulates quiescent/dormant HSCs to proliferate, leading to HSC exhaustion. Under conditions of chronic IFN stimulation including inflammation, infection, and IRF-2 deficiency, not only hematopoietic stem cells, but also cancer stem cells change from their dormant status and enter the cell cycle to proliferate. Many anticancer therapies, such as 5-FU, can disrupt proliferating cells, but not quiescent cells. Furthermore, cancer cells sometimes become resistant to drugs with molecular targets, such as imatinib. Targeting specific intrinsic IFN signaling in conjunction with the use of anticancer drugs may be a useful approach for abrogating cancer stem cells.

\section{Acknowledgements}

I thank Drs. I. Hamaguchi, K. Takizawa, T. Mizukami, M. Kuramitsu, H. Momose, and K. Yamaguchi for their experimental support and useful discussions. I wish to also thank Ms. K. Furuhata for cell sorting and Ms. M. Tsuruhara and Ms. K.Araki for molecular technical assistance. This work was supported in part the Japan Society for the Promotion of Science and the Ministry of Education, Science, Sports, and Culture of Japan.

\section{Author details}

\section{Atsuko Masumi*}

Address all correspondence to: amasumi@nih.go.jp

Department of Safety Research on Blood and Biological Products, National Institute of Infectious Diseases, Tokyo, Japan 


\section{References}

[1] Ema, H, Morita, Y, Yamazaki, S, Matsubara, A, Seita, J, Tadokoro, Y, Kondo, H, Takano, H, \& Nakauchi, H. Adult Mouse Hematopoietic Stem Cells: Purification and SingleCell Assays. Nature Protocols (2006). , 1, 2979-2987.

[2] Orkin, S. H, \& Zon, L. I. Hematopoiesis: An Evolving Paradigm for Stem Cell Biology. Cell (2008). , 132, 631-644.

[3] Arai, F, \& Suda, T. (2007). Maintenance of Quiescent Hematopoietic Stem Cells in the Osteoblastic Niche. Annals of the New York Academy of Sciences 2007; 1106, 41-53.

[4] Orford, K. W, \& Scadden, D. T. Deconstructing Stem Cell Self-Renewal: Genetic Insights into Cell-Cycle Regulation. Nature Reviews Genetics (2008). , 9, 115-128.

[5] Majeti, R, Park, C. Y, \& Weissman, I. L. Identification of a Hierarchy of Multipotent Hematopoietic Progenitors in Human Cord Blood. Cell Stem Cell (2007). , 1, 635-645.

[6] Mcdermott, S. P, Eppert, K, Lechman, E. R, Doedens, M, \& Dick, J. E. Comparison of Human Cord Blood Engraftment between Immunocompromised Mouse Strains. Blood (2010). , 116, 193-200.

[7] MacNamara KCJones M, Martin O, Winslow GM. Transient Activation of Hematopoietic Stem and Progenitor Cells by IFN-gamma during Acute Bacterial Infection. PLoS One (2011). e28669. DOI:10.1371/journal.pone.0028669.

[8] MacNamara KCOduro K, Martin O, Jones DD, McLaughlin M, Choi K, D.L. Borjesson, G.M. Winslow. Infection-induced Myelopoiesis during Intracellular Bacterial Infection is Critically Dependent Upon IFN-Gamma Signaling. Journal of Immunology 186; (2011). , 2011, 1032-1043.

[9] Scumpia, P, Kely-scumpia, K, Delano, M, Weinstein, J, Cuenca, A, Al-quran, S, Bovio, I, Akira, S, Kumagai, Y, \& Moldawer, L. Bacterial Infection Induces Hematopoietic Stem and Progenitor Cell Expansion in the Absence of TLR Signaling. Journal of Immunology (2010). , 184, 2247-2251.

[10] Singh, P, Yao, Y, Weliver, A, Broxmeyer, H, Hong, S, \& Chang, C. Vaccinia Virus Infection Modulates the Hematopoietic Cell Compartments in the Bone Marrow. Stem Cells (2008). , 26, 1009-1016.

[11] Welner, R. S, Pelayo, R, Nagai, Y, Garrett, K. P, Wuest, T. R, Carr, D. J, Borghesi, L. A, Farrar, M. A, \& Kincade, P. W. Lymphoid Precursors are directed to Produce Dendritic Cells as a Result of TLR9 Ligation during Herpes Infection. Blood (2008). , 112, 3753-3761.

[12] Kiladjian, J. J, Mesa, R. A, \& Hoffman, R. The renaissance of interferon therapy for the treatment of myeloid malignancies, Blood (2011). , 117(2011), 4706-4715. 
[13] Taniguchi, T, Ogasawara, K, Takaoka, A, \& Tanaka, N. IRF Family of Transcription Factors as Regulators of Host Defense. Annual Review Immunology (2001). , 19, 623-655.

[14] Essers, M, Offner, S, Blanco-bose, W, Waibler, Z, Kalinke, U, Duchosal, M, \& Trumpp, A. IFNa Activates Dormant Hematopoietic Stem Cells In Vivo. Nature (2009). , 458, 904-909.

[15] Masumi, A, Miyatake, S, Kohno, T, \& Matsuyama, T. Interferon Regulatory Factor-2 Regulates Hematopoietic Stem Cells in Mouse Bone Marrow. InTech-Open (2012). , 2012, 91-112.

[16] Sato, T, Onai, N, Yoshihara, H, Arai, F, Suda, T, \& Ohteki, T. Interferon Regulatory Factor-2 Protects Quiescent Hematopoietic Stem Cells from Type 1 Interferon-Dependent Exhaustion. Nature Medicine (2009). , 15, 696-701.

[17] Van Der Wath, R. C, Wilson, A, Laurenti, E, Trumpp, A, \& Lio, P. Estimating Dormant and Active Hematopoietic Stem Cell Kinetics through Extensive Modeling of Bromodeoxyuridine Label-retaining Cell Dynamics. PLoS One (2009). e6972. DOI:10.1371/ journal.pone.0006972.

[18] Goldman, J. M. Advances in CML. Clinical Advances Hematology \& Oncology (2007). , $5,270-272$.

[19] Goldman, J. M. Chronic Myeloid Leukemia Stem Cells: Now on the Run. Journal of Clinical Oncology (2009). , 27, 313-314.

[20] Feng, C. G, Weksberg, D. C, Taylor, G. A, Sher, A, Goodell, M. A, \& The, p. GTPase Lrg-47 (Irgm1) Links Host Defense and Hematopoietic Stem Cell Proliferation. Cell Stem Cell (2008). , 2, 83-89.

[21] Horwitz, M, Uzel, G, Linton, G, Miler, J, Brown, M, Malech, H, \& Holland, S. Persistent Mycobacterium Avium Infection Following Nonmyeloablative Allogenic Peripheral Blood Stem Cell Transplantation for Interferon-g Receptor-1 Deficiency. Blood (2003). , $102,2692-2694$.

[22] Hwang, J. H, Kim, S. W, Lee, H. J, Yun, H. J, Kim, S, \& Jo, D. Y. Interferon Gamma has Dual Potential in Inhibiting or Promoting Survival and Growth of Hematopoietic Progenitors: Interactions with Stromal Cell-derived Factor 1. International Journal of Hematolology (2006). , 84, 143-150.

[23] Raefsky, E. L, Platanias, L. C, Zoumbos, N. C, \& Young, N. S. Studies of Interferon as a Regulator of Hematopoietic Cell Proliferation. Journal of Immunology (1985). , 135, 2507-2512.

[24] Selleri, C, Maciejewski, J. P, Sato, T, \& Young, N. S. Interferon-gamma Constitutively Expressed in the Stromal Microenvironment of Human Marrow Cultures Mediates Potent Hematopoietic Inhibition. Blood (1996). , 87, 4149-4157.

[25] Snoeck, H. W, Van Bockstaele, D. R, Nys, G, Lenjou, M, Lardon, F, Haenen, L, Rodrigus, I, Peetermans, M. E, \& Berneman, Z. N. Interferon-gamma Selectively Inhibits Very 
Primitive CD342+CD38- and Not More Mature CD34+CD38+ Human Hematopoietic Progenitor Cells. Journal of Experimental Medicine (1994). , 180, 1177-1182.

[26] Yang, L, Dybedal, I, Bryder, D, Nilsson, L, Sitnicka, E, Sasaki, Y, \& Jacobsen, S. E. IFNgamma Negatively Modulates Self-renewal of Repopulating Human Hemopoietic Stem Cells. Journal of Immunology (2005). , 174, 752-757.

[27] Maciejewski, J, Selleri, C, Anderson, S, \& Young, N. S. Fas Antigen Expression on CD34+ Human Marrow cells is Induced by Interferon Gamma and Tumor Necrosis Factor Alpha and Potentiates Cytokine-Mediated Hematopoietic Suppression In Vitro. Blood (1995). , 85, 3183-3190.

[28] Sato, T, Selleri, C, Young, N, \& Maciejewski, J. Hematopoietic Inhibition by Interferongamma is Partially Mediated Through Interferon Regulatory Factor-1. Blood (1995). , $86,3373-3380$.

[29] Zoumbos, N. C, Djeu, J. Y, \& Young, N. S. Interferon is the Suppressor of Hematopoiesis Generated by Stimulated Lymphocytes In Vitro. Journal of Immunology (1984). , 133, 769-774.

[30] Zoumbos, N. C, Gascon, P, Djeu, J. Y, \& Young, N. S. Interferon is a Mediator of Hematopoietic Suppression in Aplastic Anemia In Vitro and Possibly In Vivo. Proceedings of the National Academy of Sciences of the United States of America (1985). , 82, 188-192.

[31] Zhao, X, Ren, G, Liang, L, Ai, P. Z, Zheng, B, Tischfield, J. A, Shi, Y, \& Shao, C. Brief Report: Interferon-gamma Induces Expansion of Lin(-)Sca-1(+)C-Kit(+) Cells. Stem Cells (2010). , 28, 122-126.

[32] Hartner, J. C, Walkley, C. R, Lu, J, \& Orkin, S. H. ADAR1 is Essential for the Maintenance of Hematopoiesis and Suppression of Interferon Signaling. Nature Immunology (2009). , 10, 109-115.

[33] Baldridge, M. T, King, K. Y, Boles, N. C, Weksberg, D. C, \& Goodell, M. A. Quiescent Haematopoietic Stem cells are Activated by IFN-gamma in Response to Chronic Infection. Nature (2010). , 465, 793-797.

[34] Belyaev, N. N, Brown, D. E, Diaz, A. I, Rae, A, Jarra, W, Thompson, J, Langhorne, J, \& Potocnik, A. J. Induction of an IL7-R(+)c-Kit(hi) Myelolymphoid Progenitor Critically Dependent on IFN-gamma Signaling during Acute Malaria. Nature Immunology (2010). , 11, 477-485.

[35] Murray, P. J, Young, R. A, \& Daley, G. Q. Hematopoietic Remodeling in Interferongamma-deficient Mice Infected with Mycobacteria. Blood (1998). , 91, 2914-2924.

[36] Kim, J. M, Oh, Y. K, Kim, Y. J, Youn, J, \& Ahn, M. J. Escherichia Coli Up-regulates Proinflammatory Cytokine Expression in Granulocyte/Macrophage Lineages of CD34 Stem Cells Via Homodimeric NF-kappaB. Clinical and Experimental Immunology (2004). , 50. 
[37] Quinton, L. J, Nelson, S, Boe, D. M, Zhang, P, Zhong, Q, Kolls, J. K, \& Bagby, G. J. The Granulocyte Colony-Stimulating Factor Response After Intrapulmonary and Systemic Bacterial Challenges. Journal of Infectious Diseases (2002). , 185, 1476-1482.

[38] Shahbazian, L. M, Quinton, L. J, Bagby, G. J, Nelson, S, Wang, G, \& Zhang, P. Escherichia Coli Pneumonia Enhances Granulopoiesis and the Mobilization of Myeloid Progenitor Cells into the Systemic Circulation. Critical Care Medicine (2004). , 32, 1740-1746.

[39] Zhang, P, Nelson, S, Bagby, G. J, \& Siggins, R. nd, Shellito JE, Welsh DA. The Lineagec-Kit+Sca-1+ Cell Response to Escherichia Coli Bacteremia in Balb/c mice. Stem Cells (2008). , 26, 1778-1786.

[40] Zheng, C, Li, L, Haak, M, Bros, B, Frank, O, Giehl, M, Fabarius, A, Schatz, M, Weisser, A, Lorentz, C, Gretz, N, Hehlmann, R, Hochhaus, A, \& Seifarth, W. Gene Expression Profiling of CD34+ Cells Identifies a Molecular Signature of Chronic Myeloid Leukemia Blast Crisis. Leukemia (2006). , 20, 1028-1034.

[41] Muraoka, K, Tsuji, K, Yoshida, M, Ebihara, Y, Yamada, K, Sui, X, Tanaka, R, \& Nakahata, T. Thrombopoietin-independent Effect of Interferon-gamma on the Proliferation of Human Megakaryocyte Progenitors. British Journal of Haematology (1997). , 98, 265-273.

[42] Tsuji-takayama, K, Tahata, H, Harashima, A, Nishida, Y, Izumi, N, Fukuda, S, Ohta, T, \& Kurimoto, M. Interferon-gamma Enhances Megakaryocyte Colony-stimulating Activity in Murine Bone Marrow Cells. Journal of Interferon \& Cytokine Research (1996). , 16, 701-708.

[43] Huang, Z, Richmond, T, Munteen, A, Barber, D, Weiss, M, \& Crispino, J. Stat1 Promotes Megakaryopoiesis Downstream of GATA-1 in Mice. Journal of Clinical Investigation (2007). , 117, 3890-3899.

[44] Masumi, A, Hamaguchi, I, Kuramitsu, M, Mizukami, T, Takizawa, K, Momose, H, Naito, S, \& Yamaguchi, K. Interferon Regulatory Factor-2 Induces Megakaryopoiesis in Mouse Bone Marrow Hematopoietic Cells. FEBS Letters (2009). , 583, 3493-3500.

[45] Masumi, A. The Role for Interferon Regulatory Factor-2 on Mouse Hematopoietic Stem Cells in an Inflammation State. Inflammation and Regeneration (2010). , 30, 531-535. 
